\title{
PGD by FISH for a Reciprocal Translocation: First Baby from India
}

\author{
Dattatray J. Naik · Prochi F. Madon • \\ Nandkishor J. Naik • Arundhati S. Athalye • \\ Firuza R. Parikh
}

Received: 29 April 2014/ Accepted: 23 May 2014/Published online: 10 July 2014

(C) Society of Fetal Medicine 2014

\begin{abstract}
A couple with a history of five early miscarriages due to a balanced reciprocal translocation $\mathrm{t}(6 ; 19)$ $(\mathrm{p} 22 ; \mathrm{q} 13.4)$ in the wife, was referred for preimplantation genetic diagnosis (PGD) by fluorescence in situ hybridization (FISH). After oocyte retrieval, nine embryos were obtained. One cell from each embryo was biopsied, fixed and subjected to FISH using centromere and subtelomere probes for chromosomes 6 and 19. Five embryos which had unbalanced translocations, were not transferred. A balanced translocation or absence of translocation was seen in three embryos, which were transferred. One embryo had an anucleate cell and subsequently arrested. A pregnancy was achieved in the first intracytoplasmic sperm injection cycle itself and resulted in the birth of a healthy baby. This is the first baby after PGD for a reciprocal translocation in India. In 2010, the team of the authors reported the first successful pregnancy after PGD for a Robertsonian translocation and a normal child was born.
\end{abstract}

Keywords Preimplantation genetic diagnosis in India . Pre-PGD workup - Recurrent spontaneous abortions . Multiple miscarriages · Balanced translocation · FISH

\section{Introduction}

One of the causes of recurrent miscarriages is the presence of a balanced translocation in the chromosomes of either the

D. J. Naik · P. F. Madon - N. J. Naik · A. S. Athalye ·

F. R. Parikh ( $\square)$

Department of Assisted Reproduction and Genetics, Jaslok

Hospital and Research Center, 15, Dr. G. Deshmukh Marg,

Mumbai 400026, India

e-mail: geneticsjaslok@gmail.com husband or wife. This can only be detected by karyotyping, as carriers of balanced translocations are phenotypically normal. Earlier, use of donor sperm or oocytes was an option to bypass the problem. With the advent of preimplantation genetic diagnosis (PGD), couples can now have their own biological offspring and avoid repeated miscarriages.

PGD involves certain additional steps in an IVF cycle. Even though natural conception is possible, the couples have to undergo assisted reproduction in order to obtain a number of embryos from which the normal ones are then selected for transfer. After suppression followed by ovarian stimulation with hormones and subsequent oocyte retrieval, a single sperm is injected in each oocyte using the intracytoplasmic sperm injection (ICSI) or the more recent intracytoplasmic morphologically-selected sperm injection (IMSI) technique. On day 3 when the embryos have reached the 6-8 cell stage, a single blastomere is biopsied from each embryo, treated with hypotonic solution and fixed on slides with Carnoy's fixative in a manner whereby the cytoplasm is removed and the nucleus is visible under the $10 \times$ objective of a phase contrast microscope [1]. Using a rapid fluorescence in situ hybridization (FISH) technique, at least three centromeric/subtelomeric probes of the chromosomes involved in the translocation are hybridized to the nuclei of the single cells. After post hybridization washing, different colored signals are visible under the fluorescence microscope. Interpretation is based on the number of signals or colored dots seen for each probe used. A cell with a balanced translocation or without a translocation will show two signals of each probe used. Any other combination of signals indicates an unbalanced translocation which generally results in a miscarriage and hence these embryos are not transferred. A maximum of three embryos are transferred in one cycle. If there are additional normal embryos, they are cryopreserved by 


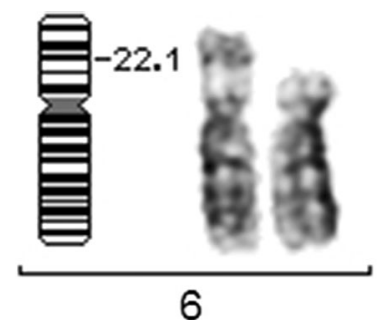

(a)

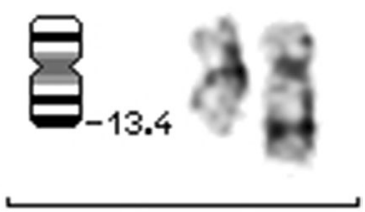

19

(b)
Fig. 1 a, b Partial karyotype from a GTG banded metaphase of the wife showing $\mathrm{t}(6 ; 19)(\mathrm{p} 22 ; \mathrm{q} 13.4)$

vitrification for later use. In India, PGD by FISH for chromosome translocations is in its infancy. Earlier, the authors reported the first successful pregnancy after PGD for a Robertsonian translocation in India [2], which resulted in the birth of a healthy baby girl. They herein report the first baby born in India after PGD for a reciprocal translocation by FISH.

\section{Case Report}

A couple from south India with a history of five early miscarriages was referred to the authors for PGD, as the wife was found to be a carrier of a balanced translocation $\mathrm{t}(6 ; 19)$ (p22;q13.4) shown in Fig. 1a, b. Karyotyping was done after most of the other causes of recurrent abortions had been ruled out. After genetic counseling, selected centromeric and sub-telomeric probes for chromosomes 6 and 19 were imported specifically for this couple prior to starting the IVF cycle. After ICSI, a total of nine embryos were available for biopsy using a diode laser. A single cell was biopsied from each embryo, labeled in separate dishes and fixed on slides after hypotonic treatment. The coordinates of the $\mathrm{X}$ and $\mathrm{Y}$ axis on the microscope were noted for each nucleus, for precise relocation after FISH. A mixture of the following probes from Abbott Molecular (Vysis) was used: TelVysion 6p (green), TelVysion 19q (orange) and CEP 6 (aqua). Co-denaturation, hybridization and posthybridization washing were done according to the manufacturer's protocol. A Zeiss fluorescence microscope, fitted with Chroma filters and Metasystems software, was used for FISH analysis. Of the nine embryos, five were abnormal with unbalanced translocations and were not transferred. The single cell of embryo 9 did not have a visible nucleus on fixation, hence biopsy of a second cell was requested, but the embryo had arrested by this time. The remaining three embryos had two signals of each color and therefore did not have the unbalanced translocation (Fig. 2a). These were subsequently tested for common aneuploidies on the same cell using the 5-color PGT probe in the second round of FISH, after washing off the signals
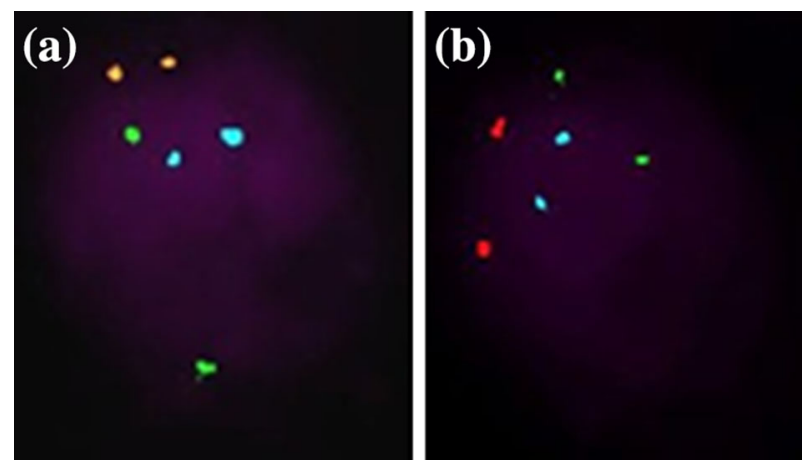

Fig. 2 a FISH on a single cell from embryo 6. Two signals of each color indicate that there is no unbalanced translocation. b FISH in round 2 on the same cell with the PGT probe shows no aneuploidy for chromosomes 13,18 and 21 . The signals of the sex chromosomes are not shown

of the first round. No aneuploidy was detected for the additional 5 chromosomes tested (Fig. 2b). The three normal embryos were developing well and were transferred by day 5. The first PGD cycle itself resulted in a pregnancy, which led to the birth of a healthy baby.

\section{Discussion}

PGD improves pregnancy outcome for translocation carriers with a history of recurrent miscarriages [3-5]. The length of time to conceive is also dramatically reduced compared with data in the literature for similar populations not undergoing PGD [6]. Studies on the impact of PGD show that it improves implantation and pregnancy rates, reduces spontaneous abortions, and improves the takehome baby rate in patients of advanced reproductive age, those with repeated IVF failures and recurrent spontaneous abortions [7, 8]. Technological advances such as array$\mathrm{CGH}$ to study chromosome abnormalities on blastocyst biopsies followed by vitrification will lead to a significant increase in implantation and pregnancy rates [9, 10]. A prePGD workup on the couple with karyotyping and FISH is very important as other subtle anomalies or signal size polymorphisms may also be detected [11, 12]. This will help in proper interpretation of FISH results.

Acknowledgments The full technical and clinical team of the Department of Assisted Reproduction and Genetics is acknowledged for their contribution. Initial support from Grants RP 318 and RP 293 of Jaslok Hospital and Research Center enabled us to establish PGD.

\section{Conflict of interest None.}

\section{References}

1. Madon PF, Athalye AS, Naik NJ, Parikh FR. Preimplantation genetic diagnosis. In: Telang $\mathbf{M}$, editor. Atlas of human assisted reproductive technologies. Delhi: Jaypee; 2007. p. 167-74. 
2. Madon PF, Athalye AS, Naik NJ, Naik DJ, Parikh FR. PGD for a Robertsonian translocation by FISH: first successful pregnancy from India. J Prenat Diagn Ther. 2010;1:20-2.

3. Otani T, Roche M, Mizuikea M, Colls P, Escudero T, Munné S. Preimplantation genetic diagnosis significantly improves the pregnancy outcome of translocation carriers with a history of recurrent miscarriage and unsuccessful pregnancies. Reprod Biomed Online. 2006;13:869-74.

4. Munné S. Preimplantation genetic diagnosis for translocations. Hum Reprod. 2006;21:839-40.

5. Lim CK, Jun JH, Min DM, Lee H-S, Kim JY, Koong MK, et al. Efficacy and clinical outcome of preimplantation genetic diagnosis using FISH for couples of reciprocal and Robertsonian translocations: the Korean experience. Prenat Diagn. 2004;24:556-61.

6. Fischer J, Colls P, Escudero T, Munné S. Preimplantation genetic diagnosis (PGD) improves pregnancy outcome for translocation carriers with a history of recurrent losses. Fertil Steril. 2010;94: 283-9.
7. Kuliev A, Verlinsky Y. Impact of preimplantation genetic diagnosis for chromosomal disorders on reproductive outcome. Reprod Biomed Online. 2008;16:9-10.

8. Harper JC, Sengupta SB. Preimplantation genetic diagnosis: state of the art 2011. Hum Genet. 2012;131:175-86.

9. Munné S, Wells D, Cohen J. Technology requirements for preimplantation genetic diagnosis to improve assisted reproduction outcomes. Fertil Steril. 2010;94:408-30.

10. Munné S. Overview of preimplantation genetic diagnosis. Expert Rev Obstet Gynecol. 2010;5:403-8.

11. Madon P. Challenges in prenatal and pre-implantation genetic diagnosis studies. Mol Cytogenet. 2014;7 Suppl 1:I50. doi:10. 1186/1755-8166-7-S1-I50

12. Madon P, Athalye A, Sanap R, Naik D, Naik N, Parikh F. Preimplantation genetic diagnosis by FISH in India: the Jaslok Hospital experience. Cytogenet Genome Res. 2014;142:226. doi: $10.1159 / 000360710$ 